\title{
Could the Material Wrapped Around the Heart Prevent Adhesion? A Case Report
}

\author{
Fatih Ayguna, b, Mehmet Ozulkua, Murat Gunday ${ }^{\mathrm{a}}$
}

\begin{abstract}
Adhesions around the heart might enhance the difficulty in performing the second heart surgery. These fibrin adhesions between epicardium and pericardium increase not only the cost, but also bleeding, duration of surgery and poor clinical outcomes. A patient, who had undergone mitral commissurotomy with the assistance of heart-lung machine (CPB) in 1998 and in whom a material that was considered to reduce adhesion had been wrapped around the heart, underwent cardiac re-surgery in 2014. No detailed information could be obtained about film barrier performed during the first surgery from the center where the surgery was performed. In our clinic, we observed the effect of the material wrapped around the heart during mitral valve replacement on adhesion.
\end{abstract}

Keywords: Heart; Cover material; Adhesion

\section{Introduction}

Adhesions around the heart might enhance the difficulty in performing second cardiac surgery. These fibrin adhesions between epicardium and pericardium increase not only the cost, but also bleeding, duration of surgery and poor clinical outcomes. If cardiac re-surgery will be performed several weeks after the first surgery, it corresponds to the time when adhesion around the heart is the most intensive and pericardial inflammation is the maximum. These materials, which are locally performed on the surgical area, made the surgeons remain distant due to their undesirable effects, mainly increased risk of infection and immune reaction [1].

Cardiac re-surgery was performed in 2014 in a patient,

Manuscript accepted for publication September 16, 2015

${ }^{a}$ Department of Cardiovascular Surgery, Konya Medical and Research Center, Baskent University, Konya, Turkey

${ }^{\mathrm{b}}$ Corresponding Author: Fatih Aygun, Department of Cardiovascular Surgery, Konya Medical and Research Center, Baskent University, Hocacihan Mah. Saray Cad No: 1, 42000 Selcuklu, Konya, Turkey.

Email: fatihaygun@ttmail.com

doi: http://dx.doi.org/10.14740/jmc2292w who had undergone mitral commissurotomy with the assistance of heart-lung machine (CPB) in 1998 and in whom a material considered to reduce adhesion has been wrapped around the heart. Detailed information on the film barrier used during the first surgery could not be obtained from the center where the surgery was performed. In our clinic, effect of the material wrapped around the heart during mitral valve replacement on adhesion was observed.

\section{Case Report}

A 64-year-old female patient presented to our clinic with shortness of breath, palpitation and limited effort. Whilst transthoracic echocardiography demonstrated mitral stenosis (mean gradient: $9.5 \mathrm{~mm} \mathrm{Hg}$, area: $1.8 \mathrm{~cm}^{2}$ ), mitral insufficiency (grade 2-3/4), pulmonary artery pressure of $60 \mathrm{~mm} \mathrm{Hg}$, and tricuspid insufficiency (grade 2-3/4), aortic valve was considered to be within the normal limits. Coronary angiography revealed no lesion. The patient was admitted to the cardiovascular surgery clinic for mitral valve replacement and tricuspid valve De Vega annuloplasty. Prior to the open heart surgery, routine laboratory analyses (complete blood count, biochemical analyses, telegram, urinalysis, dental examination report, electrocardiography, etc.) were requested, preoperative medical treatment was arranged, and the patient was prepared for surgery.

On the day of surgery, the patient was admitted to the operating room. Fentanyl, midazolam and pancuronium bromide were used for the induction of anesthesia. Standard median sternotomy was performed. Heparin sodium was given at a dose of $300 \mathrm{IU} / \mathrm{kg}$ (Nevparin ${ }^{\circledR}$ flacon $25,000 \mathrm{IU} / 5 \mathrm{~mL}$ ). CPB and cross-clamp, standard aortic and selective (VCS, $\mathrm{VCI}$ ) venous cannulation were performed. Jostra-Cobe (model 043213105 , VLC 865, Sweden) heart-lung machine was used. Crystalloid cardioplegy was given during surgery, whereas hot shot cardioplegy was given at the end of surgery. Standard left atriotomy and right atriotomy were performed. Unnecessary electrocautery use and luxury perfusion in $\mathrm{CPB}$ were avoided. Hematocrit and hemoglobin values were checked every 20 min after induction of anesthesia until the end of surgery. Intraoperative blood transfusion was performed when hematocrit value decreased to $20 \%$. Mediastinal and chest drains were placed subxyphoidally. At the end of surgery, appropriate dose of protamine hydrochloride (Protamin ${ }^{\circledR}$ ampoule 1,000 


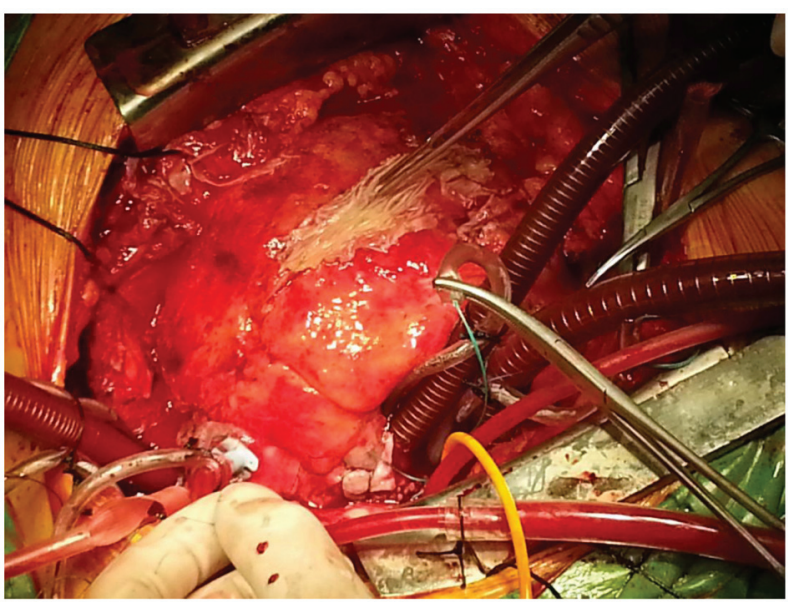

Figure 1. Wrapping material on right ventricle.

IU/1 mL) was administered for full-dose neutralization in the patient that underwent on-pump $\mathrm{CABG}$, and activated clothing time (ACT) was kept between 100 and $120 \mathrm{~s}$. The patient was then admitted to the cardiovascular surgery intensive care unit.

\section{Discussion}

Although the mechanism of adhesion formation is unclear, inflammatory response plays a role in its pathogenesis. Open heart surgery increases reactive oxygen species (ROS). This is reported to cause postoperative adhesion. In some animal experiments, it was demonstrated that ROS scavengers could prevent adhesion formation [2].

What expected after cardiac surgery is the complete healing of pericardium in the presence of membrane formation. Second, it results in formation of single-layer, flattened mesothelial cells over disappeared connective tissue. It has been determined that mesothelial cells can differentiate from multipotent cells that occurred on the collagen basis. These cells prevent adhesion by synthesizing growth factors, lubricants and extracellular matrix proteins and secreting fibrin scavenging factors [3]. Some local drugs or biologicals can prevent adhesion [4].

Although there is no protective method, postoperative pericardial adhesion significantly influences mortality and morbidity. Whilst some studies have focused on autogenous, heterogeneous and synthetic structures that could act as a barrier between epicardium and pericardium or sternum, some other studies have focused on the agents that could prevent adhesion after cardiac surgery [1-3]. Efficacy of synthetic and xenogeneic membranes has been understood [5]. However, there are reports that these membranes are associated with formation of calcification, capsule and epicardial reaction. Their effectivity has not been proven. As these structures are not transparent, they make the examination of cardiac structure difficult during surgery [6]. On the other hand, it was demonstrated that systemic anti-inflammatory drugs and topical fibrinolytic agents reduce adhesion. However, both approaches impair wound healing and unfavorably influence bleeding [7]. Inflammatory

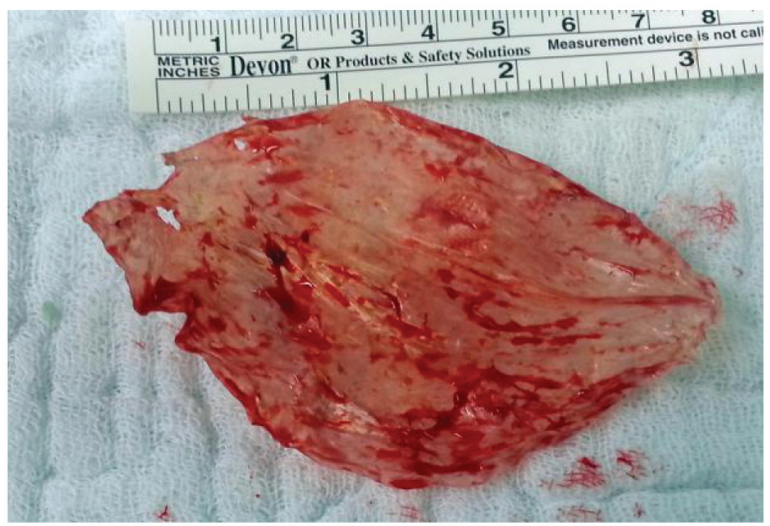

Figure 2. Wrapping material.

response is important in the pathogenesis of adhesion formation. It is known that decreased tissue oxygen due to ischemia leads to adhesion formation. Free oxygen radicals are significantly increased following endothelial tissue injury. These radicals rapidly react with oxygen and aggravate oxygen gap [2]. Free oxygen radicals such as superoxide, peroxide and hydroxyl radicals have the potential to oxidize polyunsaturated fatty acids. Oxidation of fatty acids would result in peroxidation of cell membrane lipids and accordingly in the process that would enhance vascular permeability [8]. This process causes formation of serosanguineous exudate. Exudation triggers adhesion. In general, these adhesions are exposed to lysis after $72 \mathrm{~h}$. Endogenous fibrinolytic activity is the factor that enables lysis [9]. Collected fibrin, which results from the balance between fibrin collection and degradation being in favor of fibrin, forms the adhesion [2].

It was determined that the area with adhesion-preventing material covered over the anterior and lateral aspects of the heart after sternotomy and mitral commissurotomy performed in 1998 (Fig. 1, 2) has equal adhesion (even higher in some places) to the area with no material performed while exposing to surgical procedure in 2014 . We can say that the film barrier wrapped around the heart during cardiac surgery to prevent adhesion is not effective. However, case series are needed to support this statement.

\section{References}

1. Kaushal S, Patel SK, Goh SK, Sood A, Walker BL, Backer CL. A novel combination of bioresorbable polymeric film and expanded polytetrafluoroethylene provides a protective barrier and reduces adhesions. J Thorac Cardiovasc Surg. 2011;141(3):789-795.

2. Binda MM, Molinas CR, Koninckx PR. Reactive oxygen species and adhesion formation: clinical implications in adhesion prevention. Hum Reprod. 2003;18(12):25032507.

3. Lopes JB, Dallan LA, Moreira LF, Campana Filho SP, Gutierrez PS, Lisboa LA, de Oliveira SA, et al. Synergism between keratinocyte growth factor and carboxymethyl chitosan reduces pericardial adhesions. Ann Thorac 
Surg. 2010;90(2):566-572.

4. Bel A, Kachatryan L, Bruneval P, Peyrard S, Gagnieu C, Fabiani JN, Menasche P. A new absorbable collagen membrane to reduce adhesions in cardiac surgery. Interact Cardiovasc Thorac Surg. 2010;10(2):213-216.

5. Salminen JT, Mattila IP, Puntila JT, Sairanen HI. Prevention of postoperative pericardial adhesions in children with hypoplastic left heart syndrome. Interact Cardiovasc Thorac Surg. 2011;12(2):270-272.

6. Shapira N, Gordon CI, Lemole GM. Occlusion of aortocoronary vein grafts in association with bovine pericardium. Am J Cardiovasc Pathol. 1990;3(1):87-90.
7. Wiseman DM, Kamp L, Linsky CB, Jochen RF, Pang RH, Scholz PM. Fibrinolytic drugs prevent pericardial adhesions in the rabbit. J Surg Res. 1992;53(4):362-368.

8. Kagoma P, Burger SN, Seifter E, Levenson SM, Demetriou AA. The effect of vitamin $\mathrm{E}$ on experimentally induced peritoneal adhesions in mice. Arch Surg. 1985;120(8):949-951.

9. Hellebrekers BW, Trimbos-Kemper GC, Bakkum EA, Trimbos JB, Declerck PJ, Kooistra T, Emeis JJ. Shortterm effect of surgical trauma on rat peritoneal fibrinolytic activity and its role in adhesion formation. Thromb Haemost. 2000;84(5):876-881. 